\title{
Brote familiar por el nematodo Trichostrongylus colubriformis en una zona rural de la provincia de Valdivia: una zoonosis de rara ocurrencia
}

\author{
Family outbreak caused by the nematode Trichostrongylus colubriformis in a rural area \\ of the province of Valdivia: a rare occurrence zoonoses
}

\author{
Patricio Torres H. ${ }^{1}$, Alexis Arcos B. ${ }^{2}$, Enrique Villa A. ${ }^{3}$ y Omar Cerna D. ${ }^{1}$
}

\begin{abstract}
${ }^{1}$ Instituto de Parasitología, Facultad de Medicina, Edificio de Ciencias Biomédicas, Campus Isla Teja, Valdivia, Chile.
²Unidad de Parasitología, Laboratorio Clínico, Hospital Base Valdivia, Chile.

${ }^{3}$ Policlínico del Personal, Hospital Base Valdivia, Chile.

Los autores declaran ausencia de conflicto de interés.

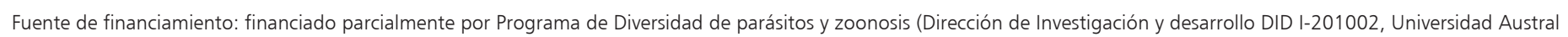
de Chile).
\end{abstract}

Recibido: 5 de noviembre de 2020 / Aceptado: 13 de abril de 2021

\section{Resumen}

La trichostrongylosis es una zoonosis de rara ocurrencia causada por nematodos intestinales, favorecida por el consumo de vegetales crudos o agua contaminada con larvas infectantes de Trichostrongylus spp. En 2015, se registró un brote familiar transmitido por alimentos en una zona rural de Valdivia, afectando a una mujer de 51 años de edad y su pareja e hija de 56 y 12 años; respectivamente. Solo la mujer adulta, el caso índice, presentó síntomas como diarrea, dolor abdominal, astenia y náuseas. Los pacientes eliminaron huevos de Trichostrongylidae gen. sp. en sus deposiciones, identificándose en dos de ellos, adultos de Trichostrongylus colubriformis. Tuvieron una buena respuesta a albendazol. En Chile, se han registrado prevalencias de infección de 0,1 a 3,5\%, incluyendo un total de 93 casos diagnosticados entre las provincias de Concepción y Llanquihue.

Palabras clave: trichostrongylosis; Trichostrongylus colubriformis; infección intestinal; enfermedades transmitidas por alimentos; zoonosis.

\section{Introducción}

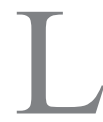

a trichostrongylidosis humana es una zoonosis de distribución cosmopolita causada por nematodos de la familia Trichostrongylidae, incluyendo especies de los géneros Trichostrongylus, Ostertagia, Haemonchus, Teladorsagia y Marshallagia ${ }^{1}$. La mayoría de los casos corresponden a infecciones por especies de Trichostrongylus

\begin{abstract}
Trichostrongylosis is a rare occurrence zoonosis caused by intestinal nematodes, favored by the consumption of raw vegetables or water contaminated with infective larvae of Trichostrongylus spp. In 2015, a family outbreak of foodborne disease was registered in a rural zone of Valdivia, affecting to 51-year-old woman and her 56-year-old partner and her 12-year-old daughter. Only the adult woman, the index case, presented symptoms such as diarrhea, abdominal pain, asthenia, and nausea. The patients revealed Trichostrongylidae gen. sp. eggs in their stools, identifying Trichostrongylus colubriformis adults in two of them. They had a good response to treatment with albendazol. In Chile, 0.1 to $3.5 \%$ prevalence of infection has been reported, including a total of 93 cases recorded between the provinces of Concepción and Llanquihue.

Keywords: trichostrongylosis; Trichostrongylus colubriformis; intestinal infection; foodborne diseases; zoonosis.
\end{abstract}

o trichostrongylosis, identificándose hasta la fecha T. affinis, T. axei, T. brevis, T. calcaratus, T. capricola, T. colubriformis, T. lerouxi, T. orientalis, T. probolurus, T. skrjabini T. vitrinus y T. longispicularis ${ }^{2}$. Las especies de Trichostrongylus viven en el intestino delgado y abomaso de animales rumiantes, equinos, cerdos, roedores lagomorfos y aves ${ }^{3}$. En Chile, de las especies mencionadas en casos humanos, se registran T. colubriformis en ovinos, llamas y alpacas; T. axei en vacunos, ovi- 
nos, caprinos, llamas y alpacas; $T$. vitrinus en vacunos, ovinos y caprinos y $T$. longispicularis en vacunos ${ }^{4}$. La trichostrongylosis humana ha sido identificada en Chile, Perú, Brasil, Uruguay, Australia, Nueva Zelanda, Estados Unidos, Italia, Francia, Rusia, Egipto, India, Armenia, Mauricio, Japón, Corea, China, Taiwán, Irán, Túnez, Zimbabue, Turquía, Iraq, Arabia Saudita, Laos y Tailandia ${ }^{2,5}$. En el ciclo vital de Trichostrongylus spp. el parásito adulto en el intestino delgado de los humanos elimina sus huevos en estado de mórula diseminándose con las deposiciones del hospedero. Los huevos al contactar con el suelo, desarrollan la larva del primer estadio que eclosiona mudando a larva del segundo y tercer estadio (L3) sucesivamente, en condiciones favorables de temperatura y humedad ${ }^{3,5}$. La infección humana se produce al consumir vegetales crudos o agua contaminada con L3 que penetran la mucosa del intestino delgado donde permanecen alrededor de 11 días. Posteriormente, las larvas regresan a la luz intestinal como adultos juveniles tras mudar dos veces, para madurar e iniciar la eliminación de huevos (período pre-patente) entre tres y cuatro semanas de adquirida la infección ${ }^{3,5}$. En el intestino delgado los parásitos causan daño traumático e inflamación en la mucosa, al fijarse por su región cefálica, dependiendo su severidad y sintomatología de la intensidad de la infección ${ }^{6}$.

Se presenta un brote familiar de tres casos de trichostrongylosis, zoonosis de rara ocurrencia manifestada como una enfermedad transmitida por los alimentos (ETA), en una zona rural de la provincia de Valdivia. Se revisan los antecedentes sobre la prevalencia de la infección en Chile.

\section{Casos clínicos y estudio parasitológico}

En abril de 2015, una mujer de 51 años de edad, residente en una zona rural, entre las localidades de Valdivia y San José de la Mariquina, acudió al policlínico del Hospital Base de Valdivia por presentar un cuadro de diarrea persistente por al menos tres semanas. Además, la paciente acusó distensión y dolor abdominal, astenia y náuseas durante la primera semana. Se le solicitó un perfil bioquímico completo y un hemograma, los que no tenían alteraciones, además de un examen parasitológico seriado de deposiciones (EPSD). Este último examen, incluyó tres muestras recolectadas día por medio y procesadas con el método de concentración por sedimentación del PAFS ("phenol, alcohol, formalin sedimentation") ", lo que implica la observación de dos preparaciones microscópicas, una en cada etapa del método ${ }^{7}$, aunque el ISP recomienda duplicar el número de preparaciones para facilitar el diagnóstico $^{8}$. Adicionalmente, se realizó un EPSD a la pareja e hija de la paciente, con 56 y 12 años de edad, ambos asintomáticos. Los resultados indicaron huevos de Trichostrongylidae gen. sp. en los tres integrantes de la familia y formas vacuoladas de Blastocystis sp. en la pareja de la primera paciente. En la paciente adulta, el caso índice, se observaron huevos en las cuatro preparaciones microscópicas del método PAFS, mientras que, en su pareja e hija solo se encontraron en una de las preparaciones. Esto refuerza la recomendación del ISP ${ }^{8}$ sobre el número de preparaciones a observar en el método. Los huevos midieron entre 87,5 y $102,5 \mu \mathrm{m}$ de largo por 37,5 a 42,5 $\mu \mathrm{m}$ de ancho, con un embrión en estado de mórula en su interior (Figura 1A). La identificación de los huevos fue realizada en el laboratorio clínico del Hospital base de Valdivia y confirmada en el Instituto de Parasitología de la Facultad de Medicina, Universidad Austral de Chile $(\mathrm{UACH})$, apoyada con material concentrado de referencia existente en la colección de dicho Instituto.

Los tres pacientes fueron tratados con una dosis única de albendazol y se les entregó un frasco con formolsalino ( $4 \%$ de formalina en $\mathrm{NaCl} 0,15 \mathrm{M}$ de $1 \%$ ) para la recolección de las deposiciones eliminadas durante las $48 \mathrm{~h}$ post-tratamiento ${ }^{6}$ para realizar la recuperación de los parásitos adultos. Solo los dos pacientes adultos devolvieron los frascos con las muestras. A los 30 días posteriores al tratamiento, ambos pacientes no tenían síntomas y al EPSD no se observaron huevos del parásito. Las deposiciones fijadas, de $48 \mathrm{~h}$ post-tratamiento, fueron depositadas por porciones en una placa de vidrio, disgregadas con agua destilada y observadas bajo microscopio estereoscópico en campana de extracción de gases. Los nematodos aislados fueron conservados en formol salino, luego colocados cada uno en un portaobjeto con dos gotas de lactofenol ${ }^{9}$ y cubiertos con un cubreobjetos. Lo anterior, permitió diafanizar los nematodos, visualizar sus características morfológicas, efectuar mediciones con micrómetro ocular e identificar a nivel de género y especie $^{3,9}$. En la paciente adulta se aislaron ocho nematodos hembras y tres machos y en su pareja se contabilizaron dos hembras y un macho. Las características de los nematodos fueron las siguientes (medidas en micras, a menos que se especifique una medida distinta, expresadas como media y mínima-máxima entre paréntesis). Machos $(\mathrm{n}=4)$ : largo corporal 5,1 (4,4-5,6) mm, ancho máximo 97,5 (90-100) (Figura 1B), en la región del poro excretor 27,7 (25-30) (Figura 1C) y en la bolsa copulatriz 123 (112,5-144) (Figura 1D); bolsa copulatriz con lóbulo dorsal reducido 40,5 (39-43) de largo, con 2 extremidades cortas y bifurcadas (Figuras 1D-E). Espículas desiguales, curvadas y de color café oscuro. Espícula derecha 130 (125-140) de largo por 22,5 (20-26) de ancho máximo; espícula izquierda 143,8 (137,5-150) de largo por 28,3 (27,5-30) de ancho máximo (Figura 1F). Gubernáculo con forma de bote 75 (73-77,5) de largo por 16,3 (15-17,5) de ancho máximo (Figura 1F). Hembras $(\mathrm{n}=5)$ : Largo corporal 5,7 (4,5$6,7) \mathrm{mm}$, ancho máximo del cuerpo 98 (90-100), ancho en las regiones del poro excretor 27,3 (25-30), vulva 88 


\section{Caso Clínico}

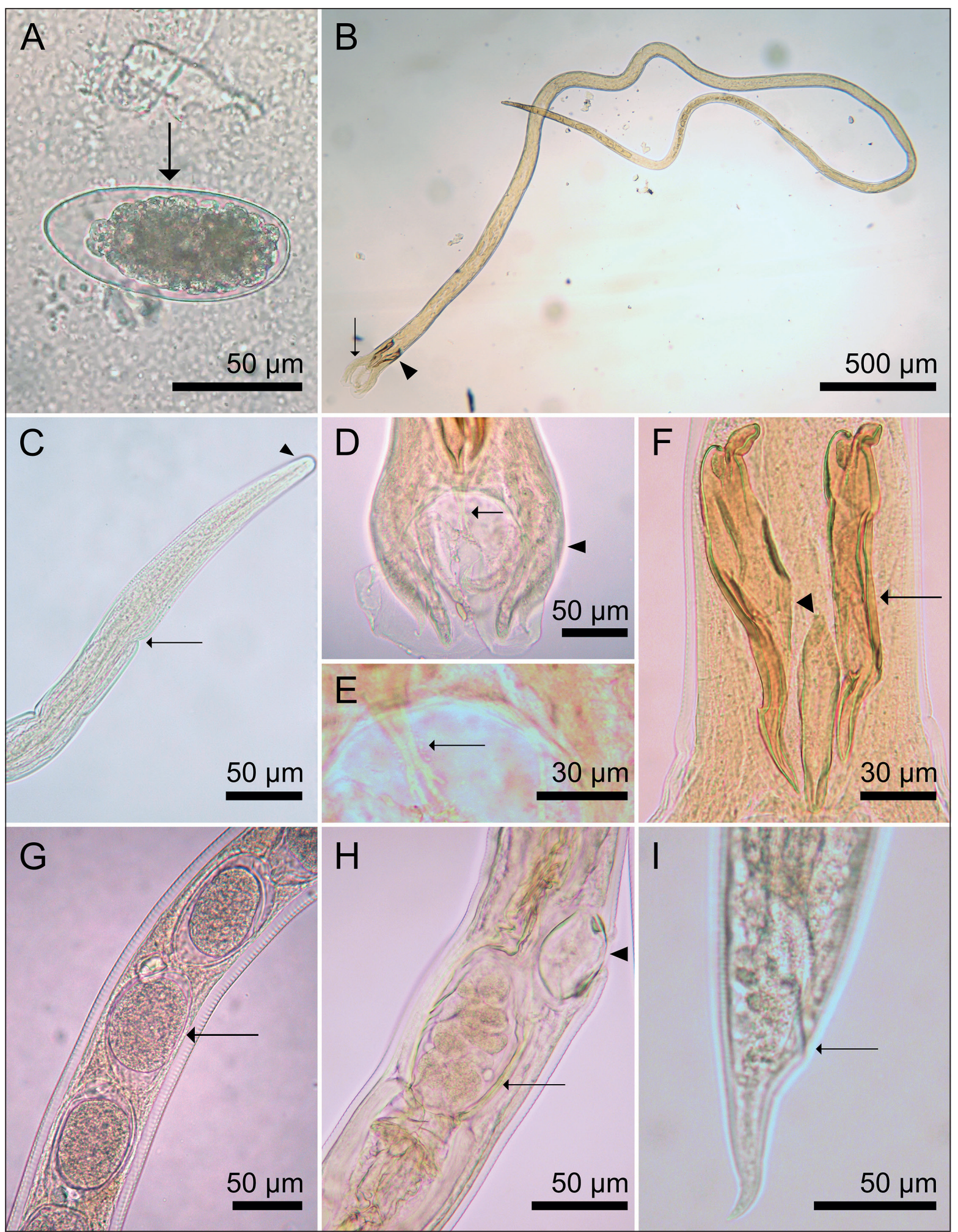

Figura 1. Características morfológicas de Trichostrongylus colubriformis. A: huevo (flecha) en las deposiciones de un paciente. B: Bolsa copulatriz (flecha) y espículas (cabeza de flecha) en la región caudal de un macho. C: Poro excretor (flecha) y región cefálica (cabeza de flecha). D: Lóbulo dorsal (flecha) de la bolsa copulatriz (cabeza de flecha). E: Ampliación del lóbulo dorsal con dos extremidades bifurcadas (flecha). F: Espículas (flecha) y gubernáculo (cabeza de flecha). G: Útero con huevos (flecha). H: Huevo en el oviyector (flecha) y vulva (cabeza de flecha). I: Ano (flecha) en una hembra. 
(75-107), y ano 36,8 (32,5-40); largo del esófago 762,3 (707-800), distancia desde la vulva (Figura $1 \mathrm{H}$ ) al extremo anterior 1,1 (1,0-1,2) mm, oviyector 417 (405-425) de largo (Figura 1H), cola (distancia entre el ano y extremo posterior) 76,6 (62,5-105) (Figura 1I). Huevos en el útero 86,6 de largo por 46,9 de ancho (83-102 de largo por 38-58 de ancho), generalmente con uno o dos extremos aguzados y un embrión en fase de mórula (Figura 1G). La identificación de los parásitos fue realizada en el Instituto de Parasitología de la Facultad de Medicina (UACH). Las características morfológicas de los parásitos adultos resultaron compatibles con $T$. colubriformis, de acuerdo a las descripciones de Levine ${ }^{3}$ y Morgan y Hawkins ${ }^{9}$, y comparaciones realizadas con material de colección preservado en el Instituto de Parasitología. Los registros fotográficos (Figura 1) fueron obtenidos mediante un microscopio Zeiss Axiostar Plus, provisto de una cámara digital Canon G6.

El brote familiar de ETA, de rara ocurrencia, fue notificado al SEREMI de salud de la región de Los Ríos.

\section{Discusión}

En el brote presentado se identificó $T$. colubriformis, causante de una zoonosis transmitida por alimentos, de rara ocurrencia, en un brote familiar de origen rural. El parásito fue identificado mediante la morfología del estado adulto recuperado después del tratamiento con albendazol en dos de los pacientes. En la menor es probable que la infección también fue causada por T. colubriformis como en sus padres, con quienes vivía y compartía alimentos. El número de parásitos hembras superó a los machos en las infecciones del presente estudio, coincidiendo con otros autores ${ }^{6,10}$. El número de nematodos en las deposiciones de la paciente con sintomatología fue mayor que en su pareja asintomática. En general, la trichostrongylosis se presenta leve o sin sintomatología y en infecciones moderadas o intensas suele asociarse a dolor abdominal, diarrea, náuseas, anemia y eosinofilia leve o pérdida de peso ${ }^{2}$. El análisis de 38 de 43 casos de trichostrongylosis en siete publicaciones de distintos países ${ }^{11-17}$ mostró manifestaciones clínicas en los siguientes porcentajes: eosinofilia leve, moderada o severa $(92,1 \%)$, dolor abdominal $(71,1 \%)$, diarrea $(57,9 \%)$, dolor gástrico $(23,1 \%)$, distensión abdominal $(21,1 \%)$ y náuseas $(10,5 \%)$, así como síntomas de menor frecuencia como flatulencia, reflujo, urticaria y mialgias $(7,9 \%)$, prurito, vómitos, hepatomegalia y esplenomegalia $(5,3 \%)$, dolor hepático, linfoadenopatía, mareos, pérdida de peso, falta de apetito, constipación y astenia (2,6\%). Los casos fueron tratados exitosamente con albendazol, pamoato de pirantel, mebendazol o ivermectina. De los 43 casos, sólo en $30(69,8 \%)$ se encontraron huevos en las heces, los que a veces fueron escasos o ausentes ${ }^{15-16}$ por discontinuidad en su eliminación, período pre-patente más prolongado (cuatro meses a dos años) o tratarse de infecciones leves 11,12. La observación de un mayor número de preparaciones microscópicas en los métodos de sedimentación o complementación con métodos de flotación es recomendable frente a la sospecha de trichostrongylosis ${ }^{5,16}$.

Los huevos de Trichostrongylus spp. pueden ser confundidos con los de otros nematodos intestinales que aunque no desarrollan sus ciclos vitales en Chile, pueden presentarse en inmigrantes de países endémicos o en chilenos que visitan tales países. Es el caso de $A n$ cylostoma spp. y Necator americanus cuyos huevos con extremos redondeados y un embrión de 4-8 blastómeros miden de 60 a $75 \mu \mathrm{m}$ de largo por 35 a $40 \mu \mathrm{m}$ de ancho ${ }^{18}$. En cambio, los huevos de Trichostrongylus spp. miden habitualmente entre 75 y $95 \mu \mathrm{m}$ de largo por 40 a $50 \mu \mathrm{m}$ de ancho ${ }^{19}$, pudiendo alcanzar 100 a $115 \mu \mathrm{m}$ de $\operatorname{largo} 0^{20}$ (Maldonado 1965), como en el presente estudio. Los huevos presentan uno, o menos frecuentemente, los dos extremos aguzados; también ambos extremos pueden presentarse redondeados pero conteniendo un embrión en estado avanzado de segmentación o mórula, a diferencia de Ancylostoma spp. y N. americanus ${ }^{21}$ (Sullivan, 2009). La existencia de microscopios debidamente calibrados, que permitan efectuar las mediciones de los huevos, es necesaria en los laboratorios clínicos para permitir el diagnóstico diferencial, como en los casos presentados ${ }^{8}$.

En raras ocasiones, en las deposiciones pueden encontrarse huevos de nematodos que parasitan plantas (Heterodera spp. y Meloidogyne spp.), los que al ser ingeridos accidentalmente al consumir vegetales crudos, y de no ser reconocidos por el microscopista, pueden llevar a errores en su identificación. Dichos huevos miden de 82 a $120 \mu \mathrm{m}$ de largo por 24 a $43 \mu \mathrm{m}$ de ancho, suelen ser ovalados y alargados con extremos redondeados, algunos asimétricos, pudiendo o no presentar una larva ${ }^{5}$.

Desde un punto de vista epidemiológico, el entorno del grupo familiar del presente estudio mostró factores de riesgo que predisponen a la transmisión de la trichostrongylosis, como la presencia de ovinos, caprinos y vacunos criados libremente y sin tratamiento antiparasitario. Además, la familia disponía de un huerto, al cual ingresaban algunos animales domésticos, cuyos productos eran habitualmente consumidos crudos. La vivienda no disponía de agua potable y para el consumo y regadío se usaba agua de pozo. De esta manera, la infección se presentó como un brote familiar de fuente común, al estar sus miembros expuestos a la presencia de animales potencialmente hospederos, consumo de hortalizas crudas del huerto familiar al que ingresaban los animales, así como la falta de agua potable en la vivienda. La trichostrongylosis humana está asociada especialmente a la tenencia de ovinos y vacunos, su uso en ganadería o faena agrícola y condiciones sanitarias deficientes ${ }^{13,17,22-23}$. El consumo de 
1976 y 1991, los 62 casos, nuevos y recopilados entre 1938 y $1972^{26}$, un caso publicado en $1985^{35}$, siete casos en $2020^{17}$ y los del presente estudio, sumando 93 casos. Sin embargo, solo en $10(10,8 \%)$ de ellos se logró la determinación específica de especie $T$. colubriformis, en uno de los cuales hubo asociación con $T$. vitrinus ${ }^{24}$; mediante la morfología del adulto ${ }^{24} \mathrm{o}$ por técnicas moleculares aplicadas en los huevos del parásito ${ }^{17}$. En los otros 83 casos, la identificación se obtuvo mediante el estudio morfológico de los huevos, lo que solo permite diagnosticar a nivel de la familia Trichostrongylidae. Según la distribución geográfica de los casos, 79 (84,9\%) correspondieron a las provincias de Valdivia y Cautín, registrándose una minoría en las provincias de Concepción (3), Arauco (1), Ñuble (1), Ranco (5), Osorno (2), y Llanquihue (1), más un caso de origen desconocido. La infección en Chile ha sido documentada en individuos de ambos sexos, entre $\operatorname{los} 4$ a 70 años de edad ${ }^{26}$.

En conclusión, la trichostrongylosis es una infección parasitaria cuyos casos se han registrado en habitantes que residen en el sur de Chile. Debe considerarse en pacientes de zonas rurales con condiciones sanitarias deficientes, tenencia de animales rumiantes y uso de sus deposiciones en el abono de huertos cuyos productos son consumidos crudos, pudiendo causar brotes familiares de ETA.

\section{Referencias bibliográficas}

1.- Ashford R W, Crewe W. The parasites of Homo sapiens. United States: Taylor and Francis, 2003. Págs. 71-4.

2.- Sharifdini M, Derakhshani S, Slizadeh S A, Ghanbarzadeh L, Mirjalali H, Mobedi I, et al. Molecular identification and phylogenetic analysis of human Trichostrongylus species from an endemic area of Iran. Acta Tropica 2017; 176: 293-99. doi: 10.1016/j.actatropica.

3.- Levine N. Nematode parasites of domestic animals and of man. United States: Burguess Publishing Company, 1968. Págs. 165-273.

4.- Alcaíno H, Gorman, T. Parásitos de los animales domésticos en Chile. Parasitol al Día 1999; 23: 33-41.

5.- Beaver P C, Jung R C, Cupp E W. Parasitología clínica. 2a Ed. España: Salvat, 1986. Págs. 2878, 312-4.

6.- Ghadirian E, Arfaa F. Present status of trichostrongyliasis in Iran. Am J Trop Med Hyg 1975; 24: 935-41. doi: 10.4269/ ajtmh.1975.24.935.

7.- Burrows R B. A new fixative and technics for the diagnosis of intestinal parasites. Am J Clin Pathol 1967; 48: 342-6.

8.- Jercic M A, Torres P. Atlas para la identificación de elementos parasitarios microscópicos en deposiciones. Chile: Instituto de Salud Pública de Chile, 2018. Págs. 85-6.

9.- Morgan B B, Hawkins P A. Veterinary helminthology. United States: Burguess Publishing Company, 1953. Págs. 133-8.

10.- Souza R P, Souza J N, Menezes J F, Alcántara L M, Soares N M, Teixeira M C A. Human infection by Trichostrongylus spp. in residents of urban areas of Salvador city, Bahia, Brazil. Biomédica 2013; 33: 439-45. doi: 10.7705/ biomedical.v33i3.770.

11.- Boreham R E, McCowan M J, Ryan A E, Allworth A M, Robson $\mathrm{J}$ M. Human trichostrongyliasis in Queensland. Pathology 1995; 27: 182-5. doi: 10.1080/00313029500169842.

12.- Ralph A, O'Sullivan M V N, Sangster N C, Walker J C. Abdominal pain and eosinophilia in suburban goat keepers-trichostrongylosis. Med J Aust 2006; 184: 467-9. doi: 10.5694/j.13265377.2006.tb00455.x.

13.- Wall E C, Bhatnagar N, Watson J, Doherty T. An unusual case of hypereosinophilia and abdominal pain: an outbreak of Trichostrongylus imported from New Zealand. J Travel Med 2011; 18: 59-60. doi: 10.1111/j.17088305.2010.00474.x.

14.- Lattès $S$, Ferté H, Delaunay P, Depaquit J, Vassallo M, Vittier M, et al. Trichostrongylus colubriformis nematode infections in humans, France. Emerg Infect Dis 2011; 17: 1301-2. doi: 10.3201/eid1707.101519.

15.- Ashrafi K, Tahbaz A, Sharifdini M, MasComa S. Familial Trichostrongylus infection misdiagnosed as acute fascioliasis. Emerg Infect Dis 2015; 21: 1869-70. doi: 10.3201/ eid2110.141392.

16.- Buonfrate D, Angheben A, Gobbi F, Mistretta M, Degani M, Bisoffi Z. Four clusters of Trichostrongylus infection diagnosed in a single center in Italy. Infection 2017; 45: 233-6. doi: 10.1007/s15010-0160957-0.

17.- Hidalgo A, Gacitúa P, Melo A, Oberg C, Herrera C, Fonseca-Salamanca F. First molecular characterization of Trichostrongylus colubriformis infection in rural patients from Chile. Acta Parasitologica 2020; 65: 790-5. doi: 10.2478/s11686-020-00206-1.

18.- Centers for Disease Control and Prevention (CDC). Parasites-hookworm [Intestinal]. USA. [Actualizado 2019 Feb.]. Fecha de acceso: abril 2021. Disponible en http://www.cdc.gov/dpdx/ hookworm/index.html.

19.- Centers for Disease Control and Prevention (CDC). Parasites-trichostrongylosis. USA. [Actualizado 2017 Dic]. Fecha de acceso: abril 2021. Disponible en: http://www.cdc.gov/dpdx/ trichostrongylosis/index.html 
20.- Maldonado J F. Helmintiasis del hombre en América. España: Editorial Científico-Médica, 1965. Págs. 87-90.

21.- Sullivan J T. A color atlas of parasitology. United States: University of San Francisco, 2009. Págs. 138-139.

22.- Youn H. Review of zoonotic parasites in medical and veterinary fields in the Republic of Korea. Korean J Parasitol 2009; 47 (Suppl): S133-S41. doi:10.3347/kjp.2009.47.S.S133.

23.- Watthanakulpanich D, Pongvongsa T, Sanguankiat S, Nuamtanong S, Maipanich W, Yoonuan T, et al. Prevalence and clinical aspects of human Trichostrongylus colubriformis infection in Lao PDR. Acta Trop. 2013; 126: 37-42. doi: http://dx.doi. org/10.1016/j.actatropica.2013.01.002.

24.- Apt W, Prudant M, Tagle I, González G. Nuevos casos de trichostrongylosis humana en Chile. Bol Chile Parasit 1967; 22: 163-5.

25.- Neghme A, Silva R. Trichostrongylidae en Chile. Bol Inf Parasit Chilenas 1952; 7: 39-40.

26.- Torres P, Figueroa L, Navarrete N. Trichostrongylosis en la provincia de Valdivia, Chile. Bol Chile Parasit 1972; 27: 52-5.
27.- Medina G A. Prevalencia de las infecciones por parásitos y comensales intestinales en manipuladores de alimentos del área urbana de Valdivia en relación a su grado de conocimientos, creencias y actitudes sobre enteroparasitosis. (Seminario de titulación, Escuela de Tecnología Médica). Valdivia, Universidad Austral de Chile; 1976.

28.- Puga S, Figueroa L, Navarrete N. Protozoos y helmintos intestinales en la población preescolar y escolar de la ciudad de Valdivia, Chile. Parasitol al Día 1991; 57-8.

29.- Torres P, Hauser M, Santibáñez J, Marín F, Gesche W, Montefusco A. 1980. Búsqueda de Diphyllobothrium y otros parásitos intestinales en la población humana y carnívoros domésticos del sector del lago Calafquén, Chile. Bol Chile Parasit 1980; 55-61.

30.- Figueroa L, Navarrete N, Franjola R, Puga S. Enteroparasitosis en la población escolar rural de la provincia de Valdivia, Chile. Bol Chile Parasit 1981; 36: 66-7.

31.- Puga S, Grob E, Krause S, Martínez R, Schild R. Infección humana por protozoos y helmintos intestinales en dos poblaciones costaneras de la provincia de Valdivia. Rev Med Chile 1980; 108: 608-11.

32.- Abello C J. Búsqueda de Diphyllobothrium spp. y otras especies de protozoos y helmintos intestinales en comunidades ribereñas de los lagos Ranco y Maihue, Chile. (Seminario de titulación, Escuela de Tecnología Médica). Valdivia, Universidad Austral de Chile; 1991.

33.- Nilo M A. Búsqueda de Diphyllobothrium spp. y otras especies de protozoos y helmintos intestinales en comunidades ribereñas de los lagos Puyehue, Rupanco y Colico, Chile. (Seminario de titulación, Escuela de Tecnología Médica). Valdivia, Universidad Austral de Chile; 1991.

34.- Mira A. Búsqueda de Diphyllobothrium spp. y otras especies de protozoos y helmintos intestinales en la población humana y animales domésticos de localidades ribereñas lacustres del sur de Chile. (Seminario de titulación, Escuela Tecnología Médica). Valdivia, Universidad Austral de Chile; 1991.

35.- Moraleda L, Franjola R, Zamorano P, Hott H. Tricuriasis masiva asociada a balantidiasis y trichostrongylosis. Rev Med Chile 1985; 113: 1004-6. 